Atıf için / For Citation: M. Düz, "Bir kaynar su reaktör modellemesinde Np ve Am yakıt çubukları için bazı nötronik değerlerin incelenmesi”, Süleyman Demirel Üniversitesi Fen Edebiyat Fakültesi Fen Dergisi, 15(1), 140-147, 2020.

\title{
Bir Kaynar Su Reaktör Modellemesinde Np ve Am Yakıt Çubukları için Bazı Nötronik Değerlerin İncelenmesi
}

\author{
Mehtap DÜZ ${ }^{* 1}$ \\ IInönü Üniversitesi, Fen Edebiyat Fakültesi, Fizik Bölümü, 44280, Malatya, Türkiye \\ *yazışllan yazar e-posta: mehtap.gunay@inonu.edu.tr
}

(Alınış / Received: 18.03.2020, Kabul / Accepted: 17.04.2020, Yayımlanma / Published: 31.05.2020)

Özet: $\mathrm{Bu}$ çalışmada Monte Carlo metodu kullanılarak üç boyutlu kaynar su reaktör (BWR) modellemesinde bazı nötronik değerler incelendi. BWR modellemesinde reaktör koru 8x8 tipinde kare kafeslere ve her kare kafes kendi içinde 7x7 tipinde küçük kare kafeslere bölünerek tasarlandı. Bu çalışmada, yakıt çubuğu olarak \% 0,02-0,1 oranlarında Neptünyum (Np) ve Amerisyum (Am) minör aktinitleri, yakıt zırhı olarak Zirkolay-2 ( $\mathrm{Zr}-2)$, soğutucu olarak su kullanılarak 7x7 tipinde küçük kare kafesler oluşturuldu. 8x8 tipinde kare kafesler içine $\mathrm{B}_{4} \mathrm{C}$ kontrol çubukları haç şeklindeki bölgede yerleştirildi. BWR modellemesinde \% $0,02-0,1$ oranlarında $\mathrm{Np}$ ve Am yakıt çubukları ve $\mathrm{Zr}$-2 yakıt zırhı için $\mathrm{k}_{\text {eff, }}$ fisyon enerjisi ve depolanan 1sınma oranı nötronik değerleri hesapland1. MCNPX-2.7.0 Monte Carlo metodu ve ENDF/BVIII.0 nükleer veri kütüphanesi kullanılarak BWR sisteminin üç boyutlu modellemesi tasarlandi.

Anahtar kelimeler: BWR, Nötronik değerler, MCNPX-2.7.0

\section{Investigation of Some Neutronic Values for Np and Am Fuel Rods in a Boiling Water Reactor Modeling}

\begin{abstract}
In this study, some neutronic values were investigated in modeling three-dimensional boiling water reactor (BWR) using Monte Carlo method. In BWR modeling, the reactor core was designed by dividing it into $8 \times 8$ type square lattices and each square lattice in itself into $7 \times 7$ type small square lattices. In this study, small square lattices of $7 \times 7$ type were created by using Neptunium $(\mathrm{Np})$ and Americium (Am) minor actinides at the rate of $0.02-0.1 \%$ as fuel rod, Zirkolay-2 (Zr-2) as fuel clad, water as coolant. $\mathrm{B}_{4} \mathrm{C}$ control rods were placed in $8 \mathrm{x} 8$ type square lattices in the cruciform area. In BWR modeling, $\mathrm{k}_{\text {eff }}$, fission energy and heat deposition rate neutronic values were calculated for $\mathrm{Np}$ and $\mathrm{Am}$ fuel rods at the rate of $0.02-0.1 \%$ and $\mathrm{Zr}-2$ fuel clad. Three-dimensional modeling of the BWR system was designed using MCNPX-2.7.0 Monte Carlo method and ENDF/B-VIII.0 nuclear data library.
\end{abstract}

Key words: BWR, Neutronic values, MCNPX-2.7.0

\section{Giriş}

1950'lerde geliştirilen hafif su reaktörleri (LWR); yakıt olarak fissil malzeme ve hem soğutma sıvısı hem de nötron moderatörü olarak normal su kullanan en yaygın fisyon reaktörleridir. Basınç esasına dayalı elektrik enerjisi üretimi için kullanılan kaynar su reaktörü (BWR) bir tür hafif su reaktörüdür. Bugün mevcut olan tüm nükleer reaktörler 
fisyon reaktörleridir ve bu reaktörlerden kalan atık; uranyum (ağırlıkça yaklaşık \% 95), plütonyum (ağırlıkça \% 1), minör aktinitler Np, Am ve Cm (ağırlıkça \% 0,1) ve fisyon ürünlerini içermektedir. Mevcut reaktörlerden kalan iyi bir enerji kaynağına ve yüksek bir radyotoksisiteye sahip olabilen bu atıklar, ileride kullanılmak üzere depolanmaktadır. Ancak yakın gelecekte ortaya çıkacak çevre ve yakıt sorunlarına çözüm olabilmesi için bu atıkların, fisyon veya nötron yakalama gibi nükleer reaksiyonlarla kararlı ve kısa ömürlü izotoplara dönüştürülmeleri gerekmektedir [1-4].

Minör aktinitler değerlidir ancak yanlış kullanılırsa tehlikeli olabilmektedir. Bu nedenle bu çalışmada, minör aktinitlerin miktarını azaltmak için \% 0,02-0,1 oranlarında $\mathrm{Np}$ ve Am yakıt çubukları kullanıldı. Strese maruz kalma ve bazı fisyon ürünlerinin aşındırıcı etkilerinin meydana gelmesi yüzünden yakıt zırhında çatlama oluşur. Reaktör ömrü ve güç üretimi için yakıt zırhının çatlaması istenmeyen bir durumdur. Minör aktinitler, reaktör ömrü, güç üretimi, yakıt zırhında çatlama ile ilgili olarak literatürde mevcut çalışmalar [1-6] bulunmaktadır. Edsinger ve Murty [5] ve Williams ve arkadaşları [6], Zr-2'nin stres seviyesinin düşük olmasından dolayı çatlakların yakıt zırhından yayılamayacağını göstermişlerdir. Bu nedenle yapılan modellemede yakıt zırhı olarak

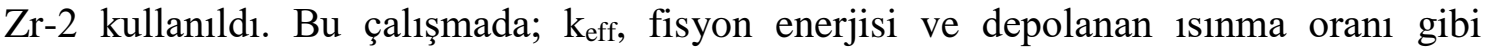
nötronik hesaplamalar yapıldı. Tasarlanan BWR sisteminde Np, Am yakıt çubuklarının ve Zr-2 yakıt zırhının nötronik hesaplamalar üzerindeki etkisi araştırıldı. Tasarlanan BWR sisteminde üç boyutlu nötronik hesaplamalar için MCNPX-2.7.0 Monte Carlo yöntemi ve ENDF/B-VIII.0 nükleer veri kütüphanesi kullanıldı.

\section{Materyal ve Metot}

\subsection{Reaktör kor geometrisi}

Tasarlanan BWR silindir şeklinde olup sistemin kor tasarımı Şekil 1'de, kor bilgileri Tablo 1'de gösterildi. Şekil 1'de görüldügü gibi, reaktör koru 8x8 kare kafese bölündü.

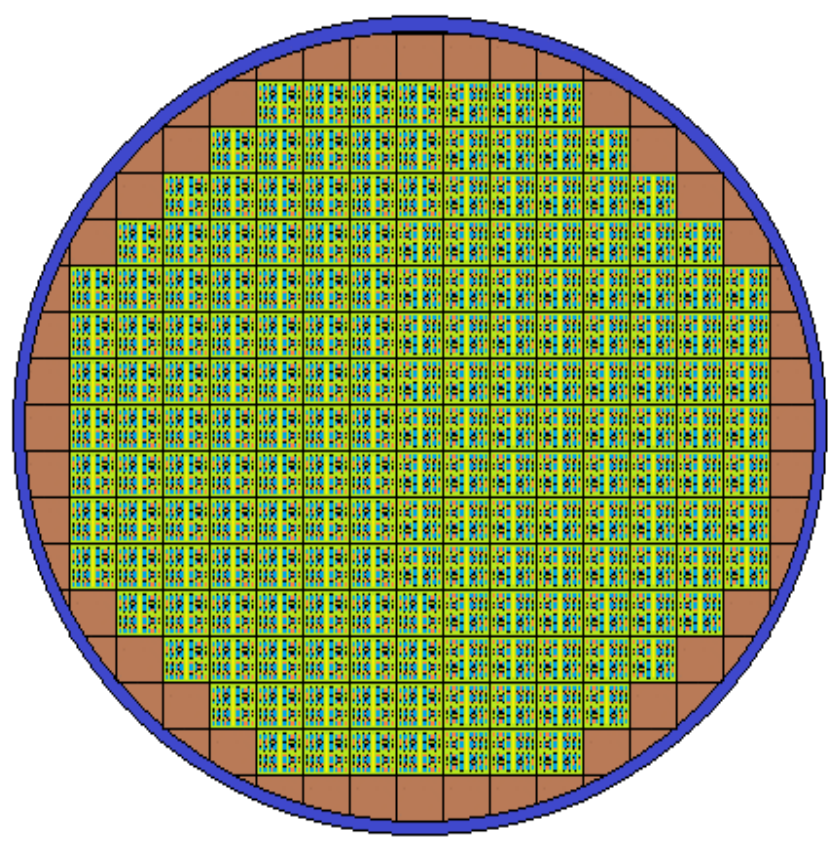

Şekil 1. BWR sisteminin kor tasarımı 
Tablo 1. BWR sisteminin kor bilgisi

\begin{tabular}{ll}
\hline Silindirin Yarıçapı $(\mathrm{cm})$ & 264,08 \\
Kor Yüksekliği $(\mathrm{cm})$ & 365,76 \\
Çelik genişliği $(\mathrm{cm})$ & 5 \\
Kare kafes genişliği $(\mathrm{cm})$ & 30,48 \\
Kare bölge genişliği $(\mathrm{cm})$ & 13,40612 \\
Küçük kare kafes genişliği $(\mathrm{cm})$ & 1,94084 \\
Yakıt çubuğu yarıçapı $(\mathrm{cm})$ & 0,60579 \\
Zırh yarıçapı $(\mathrm{cm})$ & 0,71501 \\
Boşluk genişliği $(\mathrm{cm})$ & 0,01524 \\
Toplam yakıt çubuğu sayısı & 36260 \\
Toplam haç şekilli yapı sayısı & 185 \\
Emici hücre yarıçapı $(\mathrm{cm})$ & 0,23876 \\
\hline
\end{tabular}

Reaktör korunda reflektör olarak grafit kullanıldı. Reflektörün dış kısmı SS316LN çelik ile çevrildi. Şekil 2'de gösterildiği gibi, kare kafeslerin her biri dört küçük kare bölgeye ayrıldı. Her kare bölge 7x7 tipinde küçük kare kafeslere bölündü. Küçük kare kafeslere silindirik yakıt hücreleri yerleştirildi. Reaktivite kontrolünü sağlamak için kullanılan $\mathrm{B}_{4} \mathrm{C}$ kontrol çubukları dört küçük kare kafes arasına haç biçiminde yerleştirildi. Emici hücreler silindir biçiminde olup haç şekilli yapıya yerleştirildi. Her haç şekilli yapıda toplam 84 emici hücre (kanat başına 21) kullanıldı. Böylece, tasarlanan BWR sisteminde toplam 15540 emici hücre kullanıldı. Haç şekilli yapıda yapısal malzeme olarak Tip-304 paslanmaz çelik kullanıldı.

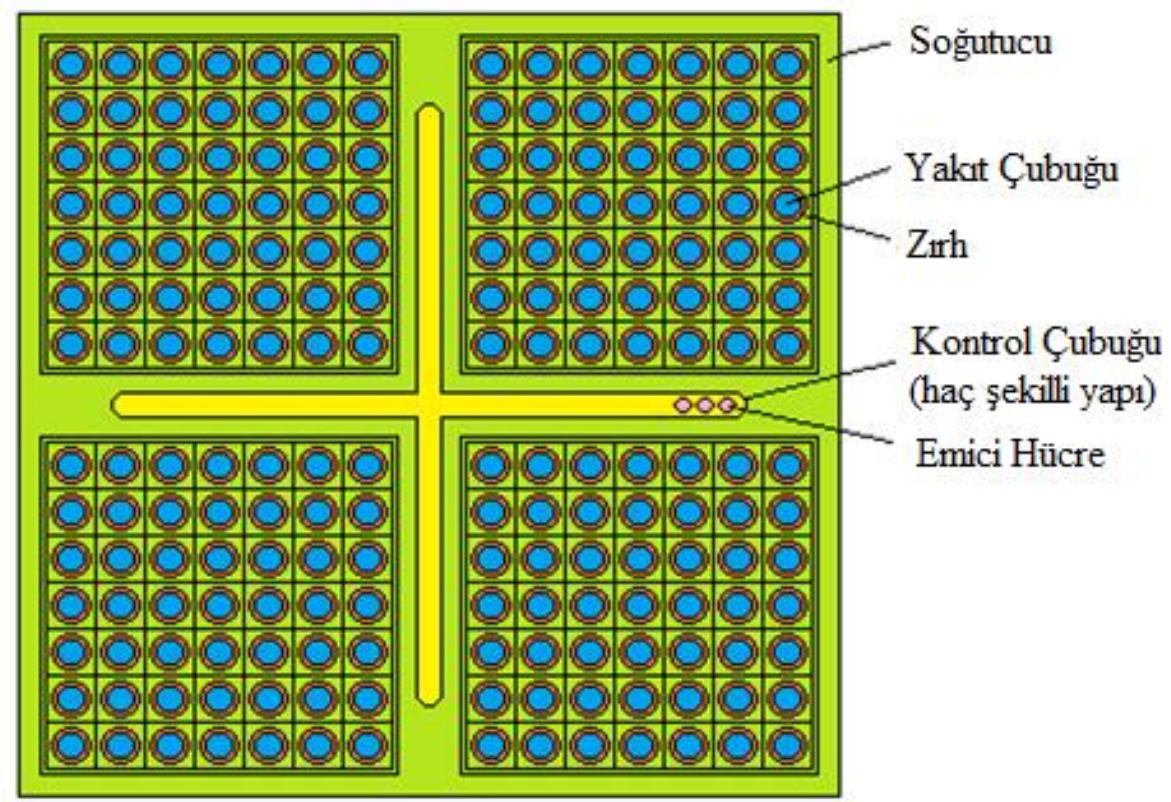

Şekil 2. BWR sistem kor tasarımında kare kafes

Yakıt hücreleri Şekil 3'te gösterildiği gibi yakıt çubuğu, boşluk ve yakıt zırhından oluşturuldu. Tasarlanan BWR sisteminde her küçük kare kafesin içine 49 yakıt çubuğu ve her kare kafesin içine 196 yakıt çubuğu yerleştirildi. Bu çalışmada, yakıt çubukları olarak \% 0,02-0,1 oranlarında $\mathrm{Np}$, Am minör aktinitleri, yakıt zırhı olarak Zr-2 kullanıldı. Tasarlanan BWR sisteminde soğutucu olarak $\mathrm{H}_{2} \mathrm{O}$ kullanıldı. 


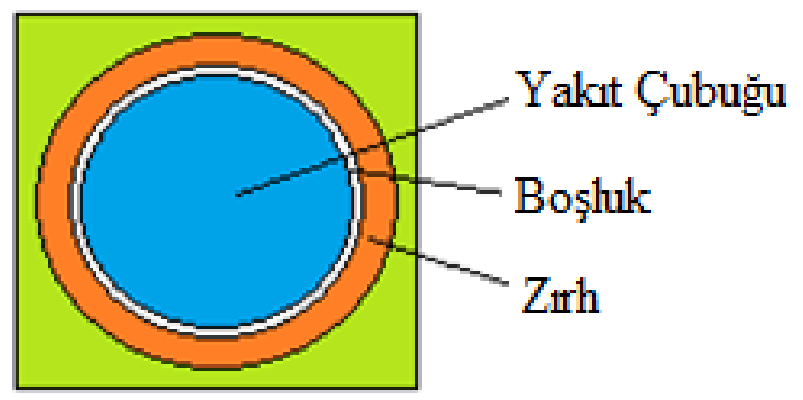

Şekil 3. Yakıt hücre geometrisi

\subsection{Sistem modelleme kodu}

Nükleer veriler, reaktör fiziği modellemesi için önemli parametrelerdir. Nükleer veriler deneysel ölçüm, teorik hesaplama ve değerlendirilmiş nükleer veri dosyaları (ENDF) ile elde edilebilir. ENDF'ler, uluslararası deneysel veri ve hesaplamaları standartlaştırmak için farklı ülkelerden yayınlanmıştır [7-11]. Uluslararası kabul görmüş ENDF'ler model hesaplamaları ile kullanılmaktadır.

Reaktör simülasyonu ve modellemesi, deterministik yönteminin birçok fiziksel problemi, malzemelerin üç boyutlu karmaşık konfigürasyonları için Monte Carlo yöntemi geliştirilmiştir. MCNPX taşıma kodu; nötron, proton ve fotonükleer etkileşimler için ENDF/B'den derlenen standart tesir kesiti kütüphanelerini kullanmaktadır. Reaktördeki nötron dağılımını belirlemek için nötron taşınması süreci araştırılmalıdır. $\mathrm{Bu}$ amaçla, Boltzmann denklemi bir reaktördeki nötronik hesaplamalarda yaygın olarak kullanılmaktadır.

$$
\frac{1}{v} \frac{\partial}{\partial t} \phi(r, \Omega, E, t)+\Omega . \nabla \phi(r, \Omega, E, t)+\sum_{t}(r, E, t) \phi(r, \Omega, E, t)=q(r, \Omega, E, t)
$$

$\frac{1}{v} \frac{\partial}{\partial t} \phi(r, \Omega, E, t)=$ Birim zamandaki nötron akısının değişimi,

$\Omega . \nabla \phi(r, \Omega, E, t)=$ Konveksiyon nedeniyle nötron kayb1,

$\sum_{t}(r, E, t) \phi(r, \Omega, E, t)=$ Nükleer reaksiyonlar nedeniyle nötron kayb1.

Denklem 1'deki $q(r, \Omega, E, t)$ terimi aşağıdaki gibi tanımlanabilir (Denklem 2):

$$
\mathrm{q}(\mathrm{r}, \Omega, \mathrm{E}, \mathrm{t})=\int_{0}^{\infty} \mathrm{dE}^{\prime} \int_{4 \pi} \mathrm{d} \Omega \sum\left(\mathrm{r}, \mathrm{E}^{\prime} \rightarrow \mathrm{E}, \Omega^{\prime} \rightarrow \Omega\right) \phi\left(\mathrm{r}, \Omega^{\prime}, \mathrm{E}^{\prime}, \mathrm{t}\right)+\mathrm{S}(\mathrm{r}, \Omega, \mathrm{E}, \mathrm{t})
$$

$\int_{0}^{\infty} d E^{\prime} \int_{4 \pi} d \Omega \sum\left(r, E^{\prime} \rightarrow E, \Omega^{\prime} \rightarrow \Omega\right) \phi\left(r, \Omega^{\prime}, E^{\prime}, t\right)=$ Saçılma yüzünden nötron akıs1 üzerine nötronların katkısı,

$S(r, \Omega, E, t)=$ Nötron kaynaklarının nötron akısı üzerine katkısı.

MCNPX-2.7.0 versiyonuna farklı intranükleer, ön denge ve buharlaşma-fisyon modelleri uygulanmıştır. Bu modeller; Bertini [12, 13] ve ISABEL [14, 15], INCL4 [16-18], CEM2k [19] ve Dresner [20], ABLA [21] olmak üzere dört fizik paketine dayanmaktadır. Bertini ve ISABEL, INCL4 paketleri Dresner ve ABLA buharlaşma fisyon kodlarıyla birleştirilebilen INC modelleridir. CEM2k ise ön denge buharlaşma adım modelidir [22, 23]. Bu çalışmada; reaktör koru ve yakıt grubu geometrisinin BWR sistemindeki üç boyutlu modellemesi ve Boltzmann denkleminin (Denklem 1, Denklem 
2) çözülmesi için MCNPX-2.7.0 Monte Carlo yöntemi ve ENDF/B-VIII.0 nükleer veri kütüphanesi kullanıldı.

\section{Bulgular}

Bu çalışmada \% 0,02-0,1 oranında $\mathrm{Np}$ ve Am yakıt çubukları ve Zr-2 yakıt zırhı için

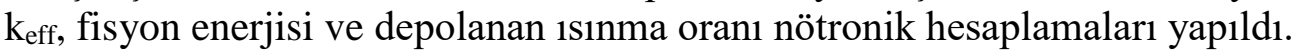

Etkin nötron çoğaltma faktörü ( $k_{\text {eff }}$ ), bir nükleer reaktörün nötron çoğalmasına nükleer reaksiyonların katkısını belirlemede etkili kritik durum olduğu için önemlidir. keff; bir nesilden diğerine nötron sayısındaki net artış olarak tanımlanır (Denklem 3). $\mathrm{k}_{\text {eff }}=1$ olması reaktörün istenen kritik çalışma modudur. Eğer $\mathrm{k}_{\mathrm{eff}}<1$ ise nötron sayıs1 katlanarak azalacaktır. Eğer $\mathrm{k}_{\text {eff }}>1$ ise, nötron sayısı katlanarak artacak ve bu da reaktörün çalıştırılması için tehlikeli olacaktır [24, 25].

$$
\mathrm{k}_{\mathrm{eff}}=\frac{\text { (bir nesilden diğerine üretilen nötron sayısı) }}{\text { (bir nesilde üretilen nötron sayısı) }}
$$

Şekil 4, \% 0,02-0,1 oranlarında Np ve Am yakıt çubukları ve Zr-2 yakıt zırhı için keff değerini göstermektedir. Şekil 4'te Zr-2 yakıt zırhı için $\mathrm{Np}$ ve Am yakıt oranlarındaki artışla keff değerinin arttığ 1 görülmektedir. Özellikle \% 0,1 oranında Np yakıtlı reaktörün istenen kritik çalışma moduna $\left(k_{\text {eff }} \cong 1\right)$ ulaştığ 1 görülmektedir.

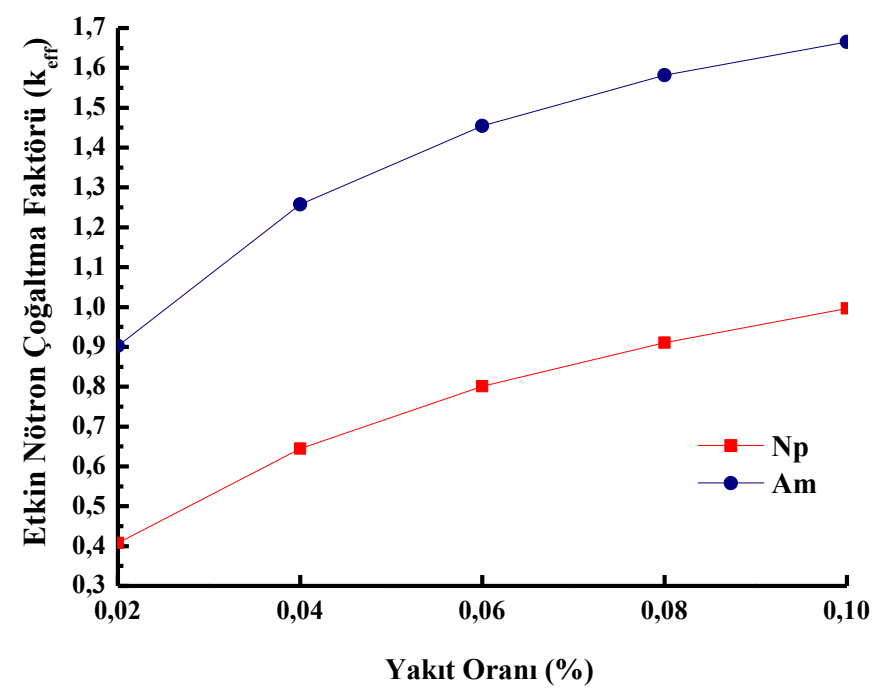

Şekil 4. BWR sisteminde Zr-2 yakıt zırhı, değişen oranlarda Np ve Am yakıtları için $k_{\text {eff }}$ değeri

Fisyon reaksiyonu tarafından salınan fisyon enerjisi; nötron yakalaması, hızlı $\gamma$-1şınları, fisyon ürünleri ve fisyon nötronlarından kinetik enerji gibi çeşitli enerji modlarını içermektedir. Bu bakımdan fisyon enerjisi, bir nükleer reaktörün nötronik hesaplamaları için önemli bir parametredir $[23,26]$. Şekil 5, \% 0,02-0,1 oranlarında Np ve Am yakıt çubukları ve Zr-2 yakıt zırhı için fisyon enerji değerini göstermektedir. Şekil 5 'te Np ve Am yakıt oranlarındaki artışla fisyon enerji değerinin arttığını görülmektedir. Şekil 5 'te fisyon enerjisinin en yüksek \% 0,1 Am yakıtından, en düşük \% 0,02 Np yakıtından kaynaklandığı görülmüştür. 


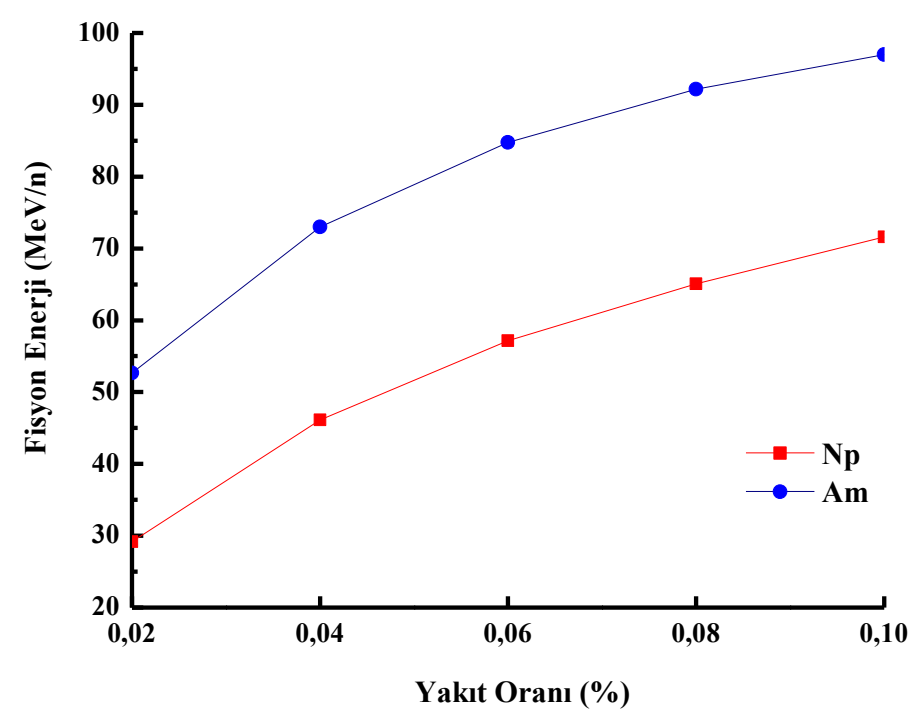

Şekil 5. BWR sisteminde Zr-2 yakıt zırhı, değişen oranlarda Np ve Am yakıtları için fisyon enerjisi

Nötron akısı, fisyon ve diğer reaksiyonlar yoluyla depolanan ısınma oranı üretilir. Bir nükleer reaktörün yakıt bölgesinde salınan fisyon enerjisinin çoğu isınmaya dönüşür. Şekil 6, \% 0,02-0,1 oranlarında $\mathrm{Np}$ ve Am yakıt çubukları ve Zr-2 yakıt zırhı için modellenen BWR sisteminin ilgili bölgelerindeki depolanan 1sınma oranı değerini göstermektedir. Şekil 6'dan, yakıt bölgesindeki yakıt oranlarının artmasıyla depolanan 1sınma oranı değerinin arttığı ve bu bölgede Am değerinin Np'dan yüksek olduğu görülmüştür. Yakıt çubuklarının etrafındaki soğutma sıvısındaki nötron ve $\gamma$-1şını radyasyonu ile küçük bir 1sı açığa çıkacaktır [27, 28]. Şekil 6, fisyon ürünleri ile nötron ve $\gamma$-1şını radyasyonu yoluyla yakıt çubuklarının etrafındaki soğutucu bölgede (su) depolanan 1sınma oranı değerinin sabit olduğunu göstermektedir. Şekil 6'dan depolanan 1sınma oranı değerine en büyük katkının yakıt bölgesinden, en küçük katkının ise yakıt zırhı bölgesinden geldiği görülmektedir.

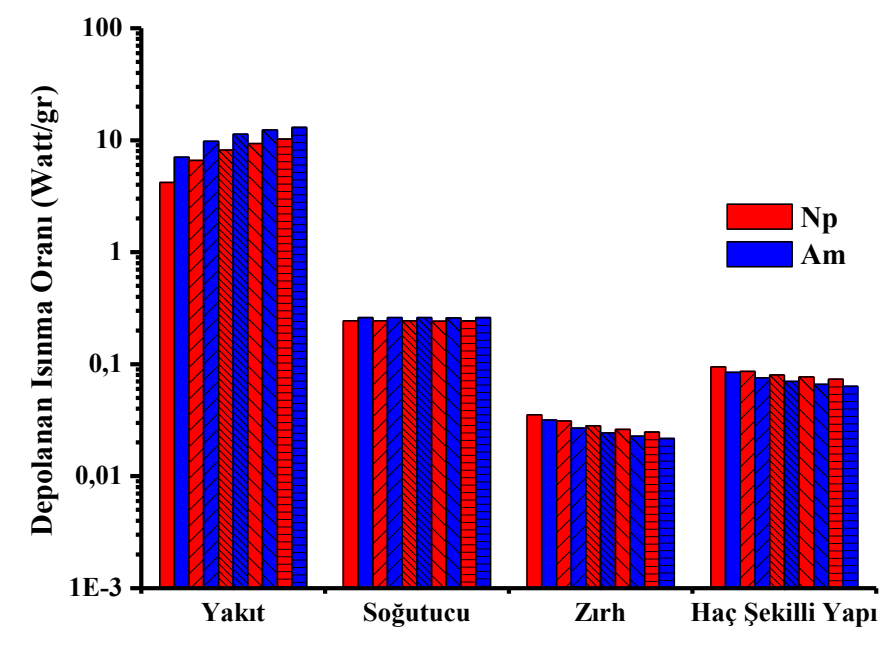

Şekil 6. Zr-2 yakıt zırhı, değişen oranlarda $\mathrm{Np}$ ve Am yakıtları $\square$ 0,02\%, $\square$, $0,04 \%$, $0,06 \%, 5 \backslash 0,08 \%$, 辰0,1\%) için BWR sisteminin ilgili bölgelerinde depolanan isınma oran1 
Tablo 2, \% 0,02-0,1 oranlarında Np ve Am yakıt çubukları ve Zr-2 yakıt zırhı için toplam depolanan 1sınma oranı değerini (Watt/gr) göstermektedir. Tablo 2'de Np ve Am yakıt oranlarındaki artışla toplam depolanan 1sınma oranı değerinin arttığ görülmektedir. Tablo 2'den, toplam depolanan 1sınma oranı değerine en küçük katkının $\% 0,02 \mathrm{~Np}$ yakıtından ve en büyük katkının \% 0,1 Am yakıtından kaynaklandığ 1 görülmektedir.

Tablo 2. Zr-2 yakıt zırhı, değişen oranlarda Np ve Am yakıtları için toplam depolanan 1sınma oranı

\begin{tabular}{ccc}
\hline Oranlar (\%) & $\mathbf{N p}$ & $\mathbf{A m}$ \\
\hline 0,02 & 1,30 & 2,12 \\
0,04 & 1,99 & 2,89 \\
0,06 & 2,44 & 3,34 \\
0,08 & 2,76 & 3,63 \\
0,1 & 3,03 & 3,81 \\
\hline
\end{tabular}

\section{Sonuç ve Yorum}

Bu çalışmada 8x8 tipinde kare kafesli bir BWR sistemi modellemesi yapıldı. Her kare kafes $7 x 7$ tipinde küçük kare kafeslere bölündü ve bu küçük kare kafesler içine Np ve Am yakıt çubukları ile Zr-2 yakıt zırhı yerleştirildi. Çalışmada; \% 0,02-0,1 Np ve Am

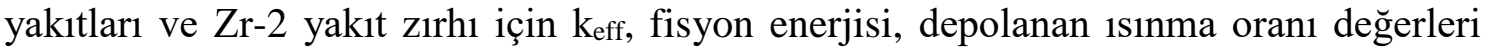
hesapland1. Tasarlanan BWR sistemindeki bu nötronik hesaplamalar MCNPX-2.7.0 Monte Carlo yöntemi ve ENDF/B-VIII.0 nükleer veri kütüphanesi kullanılarak yapıldı.

Çalışmada artan $\mathrm{Np}$ ve Am yakıt oranları ile keff, fisyon enerjisi, depolanan ısınma oranı değerlerinin arttığı gözlenmiştir. Am yakıtı ile hesaplanan nötronik sonuçların $\mathrm{Np}$ yakıtından daha yüksek olduğu bulunmuştur. Çalışmadan, \% 0,1 Am yakıtı için elde edilen nötronik sonuçların diğer tür ve oranlara göre daha iyi olduğu görülmüştür. BWR sisteminde nötronik değerlerin \% 0,1 Am yakıtı için daha iyi olması sonucu; fisyon reaktörlerinde Am yakıtının kullanılarak minör aktinit seviyesinin azalmasına avantaj sağlayabileceği öngörülmektedir.

\section{Araştırmacıların Katkı Oranı Beyanı}

Mehtap DÜZ: Araştırma, Orijinal Taslak Yazımı, Doğrulama, İnceleme ve Düzenleme, Kaynak/Materyal/Malzeme Temini, Denetim/Gözlem/Tavsiye.

\section{Destek ve Teşekkür Beyanı}

Bu çalışmanın yazarı olarak herhangi bir destek ve teşekkür beyanımın bulunmadığını bildiririm.

\section{Çatışma Beyanı}

Bu çalışmanın yazarı olarak herhangi bir çatışma beyanımın bulunmadığını bildiririm.

\section{Etik Kurul Onayı ve/veya Aydınlatılmış Onam Bilgileri}

Bu çalışmanın yazarı olarak herhangi bir etik kurul onayı ve/veya aydınlatılmış onam bilgileri beyanımın bulunmadı̆̆ını bildiririm.

\section{Kaynakça}

[1] J. Zakova, J. Wallenius, "Multirecycling of Pu, Am and Cm in BWR," Ann. Nucl. Energy, 58, 255267, 2013. 
[2] J. Loberg, M. Österlung, J. Blomgren, K. Bejmer, "Neutron detection-based void monitoring in boiling water reactors," Nucl. Sci. Eng., 164, 69-79, 2010.

[3] J. Loberg, Novel Diagnostics and Computational Methods of Neutron Fluxes in Boiling Water Reactors, Digital Comprehensive Summaries of Uppsala Dissertations from The Faculty of Science and Technology 715, ISSN: 1651-6214, 2010.

[4] R. Fridstrom, "Response of The Gamma TIP Detectors in a Nuclear Boiling Water Reactor," UPTEC F10 042, ISSN: 1401-5757, 2010.

[5] K. Edsinger, K. L. Murty, "LWR pellet-cladding interactions: materials solutions to SCC," JOM, 53, 9-13, 2001.

[6] C. D. Williams, M. Marlowe, R. B. Adamson, S. B. Wisner, R. A. Rand, J. S. Armijo, "Zircaloy-2 lined zirkonium barrier fuel cladding," Twelfth International Symposium, ASTM STP 1295, 1996, pp. 676-694.

[7] C. Wan, X. Zou, L. Cao, H. Wu, "Covariance comparisons between ENDF/B-VII.I and ENDF/BVIII.0 with application for the UAM-LWR exercises," Ann. Nucl. Energy, 138, 107-183, 2020.

[8] H. O. Wooten, "An application for streamlined and automated ENDF Cross Section Analysis and visualization (EXSAN)," Ann. Nucl. Energy, 129, 482-486, 2019.

[9] O. Kabach, A. Chetaine, A. Benchrif, "Processing of JEFF-3.3 and ENDF/B-VIII.0 and testing with critical benchmark experiments and TRIGA Mark II research reactor using MCNPX," Appl. Radiat. Isot., 150, 146-156, 2019.

[10] B. Şarer, A. Aydın, M. Günay, M. E. Korkmaz, E. Tel, "Calculations of neutron induced production cross-sections of ${ }^{180,182,183,184,186} \mathrm{~W}$ up to $20 \mathrm{MeV}, "$ Ann. Nucl. Energy, 36, 417-426, 2009.

[11] M. Günay, "Investigation of radiation damage in structural material of APEX reactor by using Monte Carlo Method," Ann. Nucl. Energy, 53, 59-63, 2013.

[12] H. W. Bertini, "Low-energy intranuclear cascade calculation," Phys. Rev., 131, 1801, 1963.

[13] H. W. Bertini, "Intranuclear-Cascade calculation of the secondary nucleon spectra from nucleonnucleus interactions in the energy range 340 to $2900 \mathrm{MeV}$ and comparisons with experiment," Phys. Rev., 188, 1711, 1969.

[14] Y. Yariv, Z. Fraenkel, "Intranuclear Cascade Calculation of High-Energy Heavy-Ion Interactions," Phys. Rev. C, 20, 2227, 1979.

[15] Y. Yariv, Z. Fraenkel, "Intranuclear Cascade Calculation of High Energy Heavy Ion Collisions: Effect of Interactions Between Cascade Particles," Phys. Rev. C, 24, 488, 1981.

[16] A. Boudard, J. Cugnon, S. Leray, C. Volant, "Intranuclear Cascade Model for a Comprehensive Description of Spallation Reaction Data," Phys. Rev. C, 66, 615, 2002.

[17] J. Cugnon, "Proton-Nucleus Interaction at High Energy," Nucl. Phys. A, 462, 751-780, 1987.

[18] J. Cugnon, C. Volant, S. Vuillier, "Improved Intranuclear Cascade Model for Nucleon-Nucleus Interactions," Nucl. Phys. A, 620, 475-509, 1997.

[19] S. G. Mashnik, V. D. Toneev, "MODEX - The Program for Calculation of The Energy Spectra of Particles Emitted in The Reactions of Pre-equilibrium and Equilibrium Statistical Decays," JINR P4-8417, 25, 1974.

[20] L. Dresner, "EVAP - a FORTRAN Program for Calculating The Evaporation of Various Particles from Excited Compound Nuclei," Report ORNL/TM-196, 1962.

[21] A. R. Junghans, M. Jong, H. G. Clerc, A. V. Ignatyuk, G. A. Kudyaev, K. H. Schmidt, "ProjectileFragment Yields as a Probe for The Collective Enhancement in The Nuclear Level Density," Nucl. Phys. A, 629, 635-655, 1998.

[22] B. Şarer, S. Şahin, M. Günay, Y. Çelik, "Comparisons of The Calculations Using Different Codes Implemented in MCNPX Monte Carlo Transport Code for Accelerator Driven System Target," Fusion Sci. Technol., 61, 302-307, 2012.

[23] M. Günay, H. Kasap, "Neutronic investigation of the application of certain plutonium-mixed fluids in a fusion-fission hybrid reactor", Ann. Nucl. Energy, 63, 432-436, 2014.

[24] S. E. Ouahdani, H. Boukhal, L. Erradi, E. Chakir, M. Azahra, T. E. Bardouni, M. Makhloul, A. Ahmed, "A temperature effect analysis of the KRITZ-1 benchmark based on keff decomposition and using the JENDL-4.0 and ENDF/B-VII.1 libraries," Prog. Nucl. Energ., 109, 121-129, 2018.

[25] J. J. Duderstadt, L. J. Hamilton, Nuclear Reactor Analysis, John Wiley\&Sons, Inc. 1976.

[26] Y. Liu, B. Kochunas, W. Martin, T. Downar, "Delayed fission energy effect on LWR normal operation and transients," Ann. Nucl. Energy, 128, 84-93, 2019.

[27] S. Şahin, B. Şarer, Y.Çelik, "Utilization of nuclear waste plutonium and thorium mixed fuel in candu reactors," Int. J. Energ. Res., 40, 1901-1907, 2016.

[28] B. Şarer, S. Şahin, Y. Çelik, M. Günay, "Evaluation of integral quantities in an accelerator driven system using different nuclear models implemented in the MCNPX Monte Carlo transport code," Ann. Nucl. Energy, 62, 382-389, 2013. 